\title{
Shear viscosity due to Landau damping from the quark-pion interaction
}

\author{
Sabyasachi Ghosh, ${ }^{1,2}$ Anirban Lahiri, ${ }^{2}$ Sarbani Majumder, ${ }^{2}$ Rajarshi Ray, ${ }^{2}$ and Sanjay K. Ghosh ${ }^{2}$ \\ ${ }^{1}$ Instituto de Fisica Teorica, Universidade Estadual Paulista, Rua Dr. Bento Teobaldo Ferraz, 271, 01140-070 Sao Paulo, Sao Paulo, Brazil \\ ${ }^{2}$ Center for Astroparticle Physics and Space Science, Bose Institute, Block EN, Sector V, Salt Lake, Kolkata 700091, India
}

(Received 23 July 2013; revised manuscript received 8 October 2013; published 3 December 2013)

\begin{abstract}
We have calculated the shear viscosity coefficient $\eta$ of the strongly interacting matter in the relaxation time approximation, where a quasiparticle description of quarks with their dynamical masses is considered from the Nambu-Jona-Lasinio model. Due to the thermodynamic scattering of quarks with pseudoscalar-type condensate (i.e., pion), a nonzero Landau damping will be acquired by the propagating quarks. This Landau damping may be obtained from the Landau cut contribution of the in-medium self-energy of a quark-pion loop, which is evaluated in the framework of real-time thermal field theory.
\end{abstract}

DOI: 10.1103/PhysRevC.88.068201

PACS number(s): 12.38.Mh, 24.85.+p

From the basic idea of the QCD asymptotic freedom at high temperatures and densities, a weakly interacting quark gluon plasma (QGP) is naturally expected to be produced in heavy ion collision (HIC) experiments. However, the experimental data from the BNL Relativistic Heavy Ion Collider (RHIC), especially the measured elliptic flow, indicate that nuclear matter is a strongly interacting liquid instead of a weakly interacting gas. The recent hydrodynamical calculations $[1,2]$ as well as some calculations of kinetic transport theory $[3,4]$ conclude that the matter produced in a HIC must have very small shear viscosity. The shear viscosity of the fluid is generally quantified by the the coefficient $\eta$ and it physically interprets the ability to transfer momentum over a distance of the mean free path. Hence the lower values of $\eta$ means the constituents of the matter interact strongly to transfer the momentum easily. Whereas a weakly interacting system must have large $\eta$ because in this case the momentum transfer between the constituents becomes strenuous.

Several theoretical attempts [5-25] have been taken to calculate the $\eta$ of the strongly interacting matter at very high [5], intermediate [6,7], and low [8-16] temperatures, where some special attention was drawn to the smallness of its original value with respect to its lower bound ( $\eta=\frac{s}{4 \pi}$, where $s$ is the entropy density), commonly known as the KSS bound [26]. A most interesting fact, which has been added with the recent theoretical understanding of $\eta$ for strongly interacting matter, is that the $\eta / s$ may reach a minimum in the vicinity of a phase transition [19-23] (see also [27]) like the liquid-gas phase transition of certain materials, e.g., nitrogen, helium, or water. These investigations demand a better understanding to zoom in on the temperature $T$ dependence of $\eta$ of the strongly interacting matter near the phase transition. Inspired by this motivation, in this Brief Report we have addressed the $\eta(T)$ due to forward and backward scattering of the quark-pion interaction.

In the relaxation time approximation, the $\eta$ of the quark [23] and pion $[15,16]$ medium (for $\mu=0$ ) can be expressed as

$$
\begin{aligned}
\eta= & \frac{8 \beta}{5} \int \frac{d^{3} \vec{k}}{(2 \pi)^{3}} \frac{\vec{k}^{4}}{\omega_{Q}^{2}} \frac{n_{Q}\left(1-n_{Q}\right)}{\Gamma_{Q}} \\
& +\frac{\beta}{5} \int \frac{d^{3} \vec{k}}{(2 \pi)^{3}} \frac{\vec{k}^{4}}{\omega_{\pi}^{2}} \frac{n_{\pi}\left(1+n_{\pi}\right)}{\Gamma_{\pi}},
\end{aligned}
$$

where $n_{Q}=\frac{1}{e^{\beta \omega} Q+1}$ and $n_{\pi}=\frac{1}{e^{\beta \omega \pi}-1}$ are, respectively, the Fermi-Dirac distribution of the quark and the Bose-Einstein distribution of the pion with $\omega_{Q}=\sqrt{\vec{k}^{2}+M_{Q}^{2}}$ and $\omega_{\pi}=$ $\sqrt{\vec{k}^{2}+m_{\pi}^{2}}$. The $\Gamma_{Q}$ and $\Gamma_{\pi}$ are Landau damping of the quark and pion, respectively. Following the quasiparticle description of the Nambu-Jona-Lasinio (NJL) model [28], the dynamical quark mass $M_{Q}$ is considered and it is generated due to the quark condensate

$$
\left\langle\bar{\psi}_{f} \psi_{f}\right\rangle=-\frac{M_{Q}-m_{Q}}{2 G}
$$

where $m_{Q}$ is the current quark mass. In the medium, the relation above becomes (for $\mu=0$ )

$$
M_{Q}=m_{Q}+4 N_{f} N_{c} G \int \frac{d^{3} \vec{k}}{(2 \pi)^{3}} \frac{M_{Q}}{\omega_{Q}}\left(1-2 n_{Q}\right) .
$$

This relation shows that the constituent quark mass tends to be the current quark mass at very high temperature where the nonzero quark condensate becomes small.

This Landau damping $\Gamma_{Q}$ and $\Gamma_{\pi}$ may be estimated from the self-energy graphs of quark and pion at finite temperature for quark-pion and quark-antiquark loops, respectively. These are, respectively, expressed as

$$
\Gamma_{Q}=-\operatorname{Im} \Sigma^{R}\left(k_{0}=\sqrt{\vec{k}^{2}+M_{Q}^{2}}, \vec{k}\right)
$$

and

$$
\Gamma_{\pi}=-\frac{1}{m_{\pi}} \operatorname{Im} \Pi^{R}\left(k_{0}=\sqrt{\vec{k}^{2}+m_{\pi}^{2}}, \vec{k}\right),
$$

where $\Sigma^{R}$ and $\Pi^{R}$ are, respectively, the retarded parts of the quark and pion self-energies at finite temperature. Their diagrammatic representations are shown in Figs. 1(a) and 1(b), respectively. Following the real-time formalism of thermal field theory, the retarded part of the inmedium quark self-energy for a quark-pion loop is given 


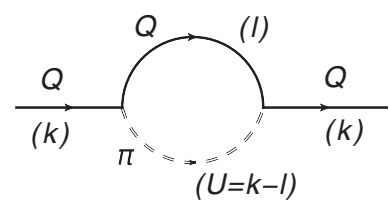

(a)

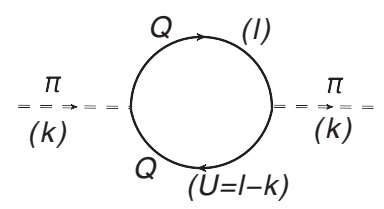

(b)
FIG. 1. Diagram of (a) quark and (b) pion self-energies for quarkpion and quark-antiquark loops, respectively.

by [29]

$$
\begin{aligned}
\Sigma^{R}\left(k_{0}, \vec{k}\right)= & \int \frac{d^{3} \vec{l}}{(2 \pi)^{3}} \frac{1}{4 \omega_{Q}^{l} \omega_{\pi}^{U}}\left[\frac{\left(1-n_{Q}^{l}\right) L_{1}^{Q}+n_{\pi}^{U} L_{3}^{Q}}{k_{0}-\omega_{Q}^{l}-\omega_{\pi}^{U}+i \eta}\right. \\
& +\frac{n_{Q}^{l} L_{1}^{Q}+n_{\pi}^{U} L_{4}^{Q}}{k_{0}-\omega_{Q}^{l}+\omega_{\pi}^{U}+i \eta}+\frac{-n_{Q}^{l} L_{2}^{Q}-n_{\pi}^{U} L_{3}^{Q}}{k_{0}+\omega_{Q}^{l}-\omega_{\pi}^{U}+i \eta} \\
& \left.+\frac{n_{Q}^{l} L_{2}^{Q}+\left(-1-n_{\pi}^{U}\right) L_{4}^{Q}}{k_{0}+\omega_{Q}^{l}+\omega_{\pi}^{U}+i \eta}\right],
\end{aligned}
$$

where $L_{i}^{Q}, i=1, \ldots, 4$ denotes the values of $L^{Q}\left(l_{0}, \vec{l}\right)$ for $l_{0}=\omega_{Q}^{l},-\omega_{Q}^{l}, k_{0}-\omega_{\pi}^{U}, k_{0}+\omega_{\pi}^{U}$, respectively, with $\omega_{Q}^{l}=$ $\sqrt{\vec{k}^{2}+M_{Q}^{2}}$ and $\omega_{\pi}^{U}=\sqrt{(\vec{k}-\vec{l})^{2}+m_{\pi}^{2}}$. Here $n_{Q}^{l}\left(\omega_{Q}^{l}\right)$ is the
Fermi-Dirac distribution function of the quark, whereas $n_{\pi}^{U}\left(\omega_{\pi}^{U}\right)$ denotes the Bose-Einstein distribution function of the $\pi$ meson.

During the extraction of the imaginary part of $\Sigma^{R}\left(k_{0}, \vec{k}\right)$, we will get four $\delta$ functions associated with the four individual terms of Eq. (6), which generate four different region in the $k_{0}$ axis where the $\operatorname{Im} \Sigma^{R}\left(k_{0}, \vec{k}\right)$ will be nonzero. From the nonzero values of $\operatorname{Im} \Sigma^{R}\left(k_{0}, \vec{k}\right)$ the region of discontinuities or branch cuts of $\Sigma^{R}\left(k_{0}, \vec{k}\right)$ can be identified. The regions coming from the first and fourth terms of Eq. (6) are, respectively, $k_{0}=$ $-\infty$ to $-\sqrt{\vec{k}^{2}+\left(m_{\pi}+M_{Q}\right)^{2}}$ and $k_{0}=\sqrt{\vec{k}^{2}+\left(m_{\pi}+M_{Q}\right)^{2}}$ to $\infty$. These are known as unitary cuts, and different kinds of forward and inverse decay processes are associated with these cut contributions [29,30]. Similarly the regions $k_{0}=$ $-\sqrt{\vec{k}^{2}+\left(m_{\pi}-M_{Q}\right)^{2}}$ to 0 , and $k_{0}=0$ to $\sqrt{\vec{k}^{2}+\left(m_{\pi}-M_{Q}\right)^{2}}$ are coming from the second and third terms, respectively. These purely medium-dependent cuts are known as Landau cuts, and different kinds of forward and inverse scattering processes are physically interpreted by these cut contributions $[29,30]$. So the third term of $\operatorname{Im} \Sigma^{R}\left(k_{0}, \vec{k}\right)$ at the on-shell mass $k_{0}=\sqrt{\vec{k}^{2}+M_{Q}^{2}}, \vec{k}$ of the quark is responsible for the Landau damping $\Gamma_{Q}$ and it is given by [29]

$$
\Gamma_{Q}=-\operatorname{Im} \Sigma^{R}\left(k_{0}=\sqrt{\vec{k}^{2}+M_{Q}^{2}}, \vec{k}\right)=\left[\int \frac{d^{3} \vec{l}}{(2 \pi)^{3}} \frac{L_{2}^{Q}}{4 \omega_{Q}^{l} \omega_{\pi}^{U}}\left(n_{Q}^{l}+n_{\pi}^{U}\right) \delta\left(k_{0}+\omega_{Q}^{l}-\omega_{\pi}^{U}\right)\right]_{k_{0}=\sqrt{\vec{k}^{2}+M_{Q}^{2}}} .
$$

Rearranging the statistical weight factor by

$$
\left(n_{Q}^{l}+n_{\pi}^{U}\right)=n_{Q}^{l}\left(1+n_{\pi}^{U}\right)+n_{\pi}^{U}\left(1-n_{Q}^{l}\right),
$$

we can find thermalized $\pi$ and $\bar{u}$ with Bose-enhanced probability $\left(1+n_{\pi}^{U}\right)$ and Pauli-blocked probability $\left(1-n_{Q}^{l}\right)$, respectively. With the help of Eq. (8), the physical significance of the Landau cut contribution may be expressed as follows. During the propagation of the $u$ quark, it may absorb the thermalized $\bar{u}$ from the heat bath and create a thermalized $\pi$ in the bath [indicated by the second part of Eq. (8)]. Again the thermalized $\pi$ may be absorbed by the medium and create the thermalized $\bar{u}$ along with a propagating $u$, which is slightly off-equilibrium with the medium [indicated by the first part of Eq. (8)].

To calculate $L_{i}^{Q}$ from the quark-pion interaction, let us start with the free Lagrangian of quarks and demand the invariance properties of the Lagrangian under chiral transformation,

$$
\psi_{f}^{\prime}=\exp \left(\frac{i \vec{\pi} \cdot \vec{\tau} \gamma^{5}}{2 F_{\pi}}\right) \psi_{f}
$$

where the chiral angle is associated with the pion field $\vec{\pi}$ and $F_{\pi}$ is the pion decay constant. Expanding up to first order of the pion field, we obtain the quark-pion interaction term $[31,32]$

$$
\begin{aligned}
\mathcal{L}_{\pi Q Q} & =\frac{-i M_{Q}}{F_{\pi}} \bar{\psi}_{f} \vec{\pi} \cdot \vec{\tau} \gamma^{5} \psi_{f} \\
& =\frac{-i M_{Q} \gamma^{5}}{F_{\pi}}(\bar{u} \bar{d})\left(\begin{array}{c}
\pi^{0} \sqrt{2} \pi^{+} \\
\sqrt{2} \pi^{-} \pi^{0}
\end{array}\right)\left(\begin{array}{l}
u \\
d
\end{array}\right) .
\end{aligned}
$$

Because we are interested in calculating the one-loop selfenergy $\Sigma^{R}$ of any quark flavor $u$ (say), we have to consider two possible loops: $u \pi^{0}$ and $d \pi^{+}$. Thanks to the isospin symmetry consideration in the Lagrangian, we can evaluate any one of the loops, say, the $u \pi^{0}$ loop, and then we have to multiply it by an isospin factor

$$
I_{F}=(1)^{2}+(\sqrt{2})^{2}=3 .
$$

From the interaction part,

$$
\mathcal{L}_{\pi^{0} u u}=-i g_{\pi Q Q} \bar{u} \gamma_{5} \pi^{0} u, \quad \text { with } \quad g_{\pi Q Q}=\frac{M_{Q}}{F_{\pi}},
$$

we can calculate $L_{a b}^{Q}\left(l_{0}, \vec{l}\right)=-I_{F} g_{\pi Q Q}^{2}\left(l-m_{l}\right)_{a b}$, where $a, b$ are Dirac indices. For simplification we have taken the scalar part only, i.e., $L^{Q}\left(l_{0}, \vec{l}\right)=I_{F} g_{\pi Q Q}^{2} m_{l}$. We have taken the parameters $m_{Q}=0.0056 \mathrm{GeV}, \bar{M}_{Q}=0.4 \mathrm{GeV}$ (for $T=$ 0 ), the three-momentum cutoff $\Lambda=0.588 \mathrm{GeV}$, and the corresponding $T_{c}=0.222 \mathrm{GeV}$ for $\mu=0$ [28].

Similar to Eq. (6), the pion self-energy $\Pi^{R}$ for the quarkantiquark loop is also received in a similar kind of form, except 
that the quantities $n_{\pi}^{U}, \omega_{\pi}^{U}=\sqrt{(\vec{k}-\vec{l})^{2}+m_{\pi}^{2}}, U=k-l$, and $L_{i}^{Q}$ are changed to $-n_{Q}^{U}, \omega_{Q}^{U}=\sqrt{(\vec{l}-\vec{k})^{2}+m_{Q}^{2}}, U=-k+l$, and $L_{i}^{\pi}$, respectively $[33,34]$. The pion on-shell mass point $k_{0}=\sqrt{\vec{k}^{2}+m_{\pi}^{2}}, \vec{k}$ will be inside the unitary cut region $k_{0}=$ $\sqrt{\vec{k}^{2}+\left(M_{Q}+M_{Q}\right)^{2}}$ to $\infty$ of $\Pi^{R}$, therefore,

$$
\Gamma_{\pi}=\frac{-\operatorname{Im} \Pi^{R}\left(k_{0}=\sqrt{\vec{k}^{2}+m_{\pi}^{2}}, \vec{k}\right)}{m_{\pi}}=\frac{-1}{m_{\pi}}\left[\int \frac{d^{3} \vec{l}}{(2 \pi)^{3}} \frac{L_{1}^{\pi}}{4 \omega_{Q}^{l} \omega_{Q}^{U}}\left(1-n_{Q}^{l}-n_{Q}^{U}\right) \delta\left(k_{0}+\omega_{Q}^{l}-\omega_{Q}^{U}\right)\right]_{k_{0}=\sqrt{\hat{k}^{2}+m_{\pi}^{2}}},
$$

where $L^{\pi}=4 I_{F} g_{\pi Q Q}^{2}\left[M_{Q}^{2}-l^{2}-k l\right]$ can be obtained from Eq. (12).

In Fig. 2, we can see the temperature dependency of Landau damping $\Gamma$ (upper panel) and collision time $\tau=\frac{1}{\Gamma}$ (lower panel) of the quark (dotted line) and pion (dashed line) for their momentum $\vec{k}=0$. Owing to the on-shell condition, the $\Gamma_{Q}$ and $\Gamma_{\pi}$ receive the nonzero values only in the temperature range where $m_{\pi}>2 M_{Q}$, which is clearly seen from the dotted and dashed lines. Corresponding nondivergent collisional times are also achieved by them in the same temperature domain. Due to the decay width of $\pi \rightarrow Q \bar{Q}$, it will more realistic to consider the pion resonance of finite width in Eq. (7). The pion spectral function due to the $Q \bar{Q}$ width may be defined as

$$
A_{\pi}(M)=\frac{1}{\pi} \operatorname{Im}\left[\frac{1}{M^{2}-m_{\pi}^{2}+i \operatorname{Im} \Pi_{\mathrm{vac}}^{R}\left(k_{0}, \vec{k}\right)}\right],
$$

where $\operatorname{Im} \Pi_{\mathrm{vac}}^{R}\left(k_{0}, \vec{k}\right)$ is the vacuum part of $\operatorname{Im} \Pi^{R}\left(k_{0}, \vec{k}\right)$ and $M=\sqrt{k_{0}^{2}-\vec{k}^{2}}$. The variation of $\operatorname{Im} \Pi^{R}(M) / m_{\pi}$ and $A_{\pi}$ with $M$ for two different temperatures are shown in Fig. 3. Replacing $m_{\pi}$ of $\Gamma_{Q}$ in Eq. (7) by $M$ and then convoluting or folding it by $A_{\pi}(M)$, we have $[33,34]$

$$
\Gamma_{Q}\left(m_{\pi}\right)=\frac{1}{N_{\pi}} \int \Gamma_{Q}(M) A_{\pi}(M) d M^{2},
$$

where $N_{\pi}=\int A_{\pi}(M) d M^{2}$. One should notice that in the narrow width approximation, i.e., for $\operatorname{Im} \Pi_{\mathrm{vac}}^{R} \rightarrow 0$, Eq. (15) is

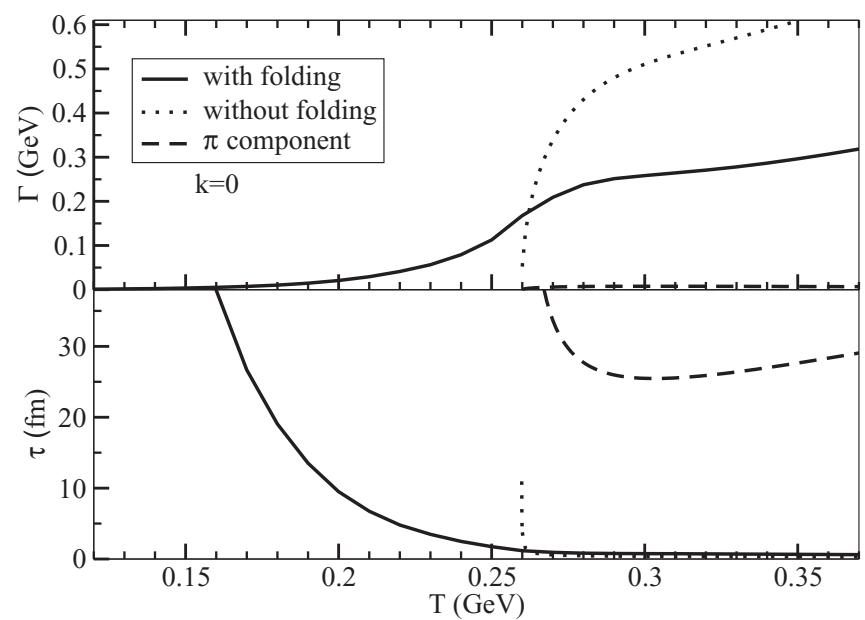

FIG. 2. Upper panel: $T$ dependency of $\Gamma_{Q}$ with (solid line) and without (dotted line) folding by $A_{\pi}$ and $\Gamma_{\pi}$ (dashed line). Lower panel: variation of corresponding collision time $\tau$ with temperature. merged to (7). The $T$ dependency of $\Gamma_{Q}$ and its corresponding $\tau$ after folding are shown by solid lines in Fig. 2. Due to folding, $\Gamma_{Q}$ at the low $T$ domain (where $m_{\pi}<2 M_{Q}$ ) has acquired some nonzero values from its vanishing contributions and at the same time the corresponding $\tau$ recovers from its divergence up to the approximate freeze-out temperature $(T \sim 120$ $150 \mathrm{GeV}$ ) of the strongly interacting matter.

By using $\Gamma_{Q}(T, \vec{k})$ from Eqs. (7) and (15) in the quark component (first term) of Eq. (1), we get the results of shear viscosity as a function of $T$, which are, respectively, described by the dotted and solid lines of Fig. 4. Being proportional to collisional time, the divergence of $\eta$ is removed after folding in those temperature regions, where $m_{\pi}<2 M_{Q}$. The contribution of $\eta$ due to $\Gamma_{\pi}(T, \vec{k})$ from Eq. (13) is shown by a dashed line in Fig. 4. After a similar kind of folding as done in Eq. (15), an almost negligible $\left(\sim 10^{-} 5 \mathrm{GeV}^{3}\right)$ contribution of $\eta$ for the pion component can be obtained, which is not included in the final results.

In the low-temperature region, $\eta$ is decreasing with increas$\operatorname{ing} T$, which is analogous to the behavior of liquid. (From our daily life experience, we see that cooking oil behaves like a less viscous medium when it is heated.) Whereas in the high-temperature domain, $\eta$ becomes an increasing function of $T$ just like a system of gas.

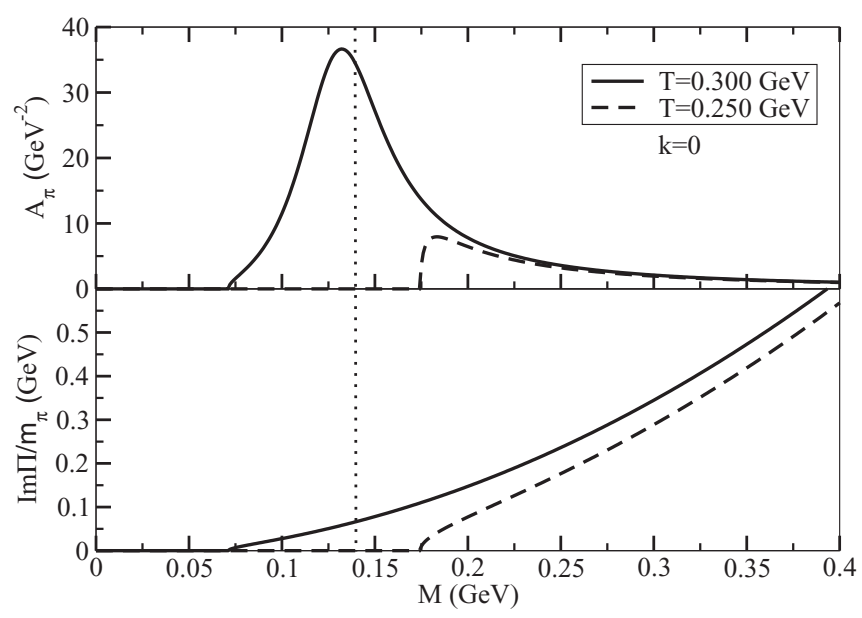

FIG. 3. Lower panel: $M$ dependency of the imaginary part of pion self-energy for the $Q \bar{Q}$ loop, which is normalized after dividing by $m_{\pi}$. Upper panel: invariant mass distribution of pion spectral function due to its $Q \bar{Q}$ width. Dotted line indicates the position of the pion pole. 


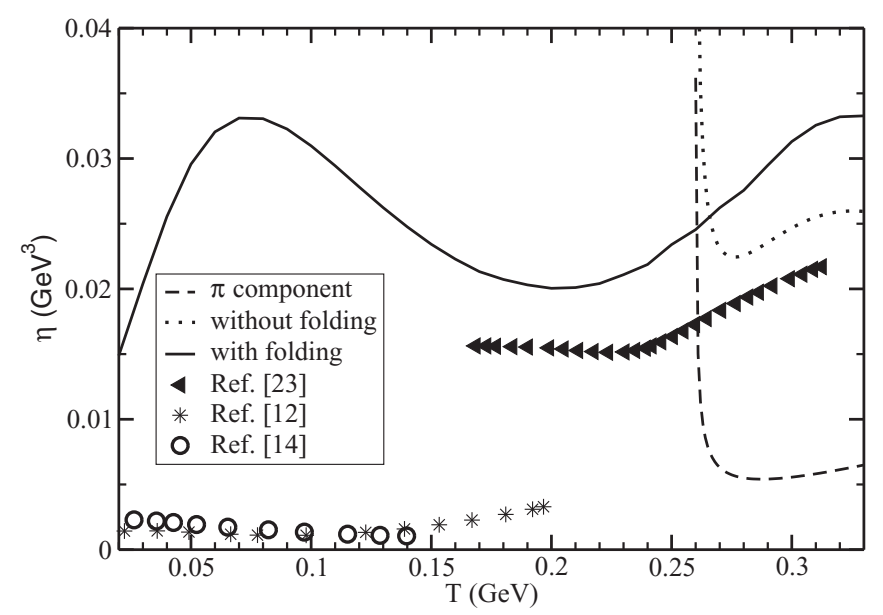

FIG. 4. Temperature dependence of $\eta$ due to $\Gamma_{\pi}$ (dashed line), $\Gamma_{Q}$ without (dotted line) and with (solid line) folding are separately shown. The results of Ref. [23] are attached to compare with our results (solid line). Also the results of the hadronic domain from Refs. [12,14] are shown.

The magnitude of $\eta$ in our approach is very close to the results of Sasaki and Redlich [23] (indicated by triangles) but is underestimated with respect to the earlier estimation in the NJL model by Zhuang et al. [22]. The lattice QCD calculation of $\eta\left(\eta \sim 0.054-0.47 \mathrm{GeV}^{3}\right.$ near $\left.T_{c}\right)$ by Meyer [7] is higher than all of these calculations. From the solid line in the lower panel of Fig. 2, we see that $\tau$ below $T \sim 160 \mathrm{MeV}$ exceeds the typical value for the time period $(\sim 30-50 \mathrm{fm})$ during which strongly interacting matter survives in the laboratories of heavy ion collisions. Therefore the estimation of $\eta$ in the low-temperature domain is quite higher than the standard calculations of $\eta$ of hadronic matter $[12,14,16]$. The earlier calculations of the NJL model [22,23] also displayed these discrepancies in the hadronic temperature domain.

In summary we have investigated the shear viscosity of strongly interacting matter in the relaxation time approximation, where a quark with its dynamical mass may have some nonzero Landau damping because of its various forward and inverse scattering with pions. This Landau damping can be obtained from the thermal field theoretical calculation of quark self-energy for a quark-pion loop. The temperature dependency of shear viscosity is coming from the thermal distribution functions, the temperature dependence of Landau damping, as well as the constituent quark mass, supplied by the temperature-dependent gap equation in the NJL model. Due to this gap equation, this constituent quark mass drops rapidly towards its current mass near $T_{c}$ to restore the chiral symmetry. A nontrivial influence of all these temperature dependencies on $\eta(T)$ is displayed in our results.

S.G. thanks Saurav Sarkar, Tamal K. Mukherjee, Soumitra Maity, Ramaprasad Adak, Kinkar Saha, and Sudipa Upadhaya for discussions that had direct and indirect influence on this work. The work of S.G. is financed by Fundação de Amparo à Pesquisa do Estado de São Paulo, FAPESP, under Contract No. 2012/16766-0.
[1] P. Romatschke and U. Romatschke, Phys. Rev. Lett. 99, 172301 (2007); M. Luzum and P. Romatschke, Phys. Rev. C 78, 034915 (2008).

[2] H. Song and U. W. Heinz, Phys. Lett. B 658, 279 (2008); Phys. Rev. C 78, 024902 (2008).

[3] Z. Xu, C. Greiner, and H. Stocker, Phys. Rev. Lett. 101, 082302 (2008); Z. Xu and C. Greiner, Phys. Rev. C 79, 014904 (2009).

[4] G. Ferini, M. Colonna, M. Di Toro, and V. Greco, Phys. Lett. B 670, 325 (2009); V. Greco, M. Colonna, M. Di Toro, and G. Ferini, Prog. Part. Nucl. Phys. 65, 562 (2009).

[5] P. B. Arnold, G. D. Moore, and L. G. Yaffe, J. High Energy Phys. 11 (2000) 001; 05 (2003) 051.

[6] A. Nakamura and S. Sakai, Phys. Rev. Lett. 94, 072305 (2005).

[7] H. B. Meyer, Phys. Rev. D 76, 101701 (2007); 82, 054504 (2010).

[8] M. Prakash, M. Prakash, R. Venugopalan, and G. Welke, Phys. Rep. 227, 321 (1993).

[9] A. Dobado and S. N. Santalla, Phys. Rev. D 65, 096011 (2002); A. Dobado and F. J. Llanes-Estrada, ibid. 69, 116004 (2004).

[10] J. W. Chen, Y. H. Li, Y. F. Liu, and E. Nakano, Phys. Rev. D 76, 114011 (2007); E. Nakano, arXiv:hep-ph/0612255.

[11] K. Itakura, O. Morimatsu, and H. Otomo, Phys. Rev. D 77, 014014 (2008).

[12] D. Fernandez-Fraile and A. Gomez Nicola, Eur. Phys. J. C 62, 37 (2009); Eur. Phys. J. A 31, 848 (2007); Int. J. Mod. Phys. E 16, 3010 (2007).

[13] A. Muronga, Phys. Rev. C 69, 044901 (2004).
[14] R. Lang, N. Kaiser, and W. Weise, Eur. Phys. J. A 48, 109 (2012).

[15] S. Gavin, Nucl. Phys. A 435, 826 (1985).

[16] S. Mitra, S. Ghosh, and S. Sarkar, Phys. Rev. C 85, 064917 (2012); S. Sarkar, Adv. High Energy Phys. 2013, 627137 (2013).

[17] S. Plumari, A. Puglisi, F. Scardina, and V. Greco, Phys. Rev. C 86, 054902 (2012).

[18] J. Peralta-Ramos and G. Krein, Int. J. Mod. Phys. Conf. Ser. 18, 204 (2012); Phys. Rev. C 84, 044904 (2011).

[19] L. P. Csernai, J. I. Kapusta, and L. D. McLerran, Phys. Rev. Lett. 97, 152303 (2006); J. I. Kapusta, arXiv:0809.3746.

[20] P. Chakraborty and J. I. Kapusta, Phys. Rev. C 83, 014906 (2011).

[21] T. Hirano and M. Gyulassy, Nucl. Phys. A 769, 71 (2006).

[22] P. Zhuang, J. Hufner, S. P. Klevansky, and L. Neise, Phys. Rev. D 51, 3728 (1995); P. Rehberg, S. P. Klevansky, and J. Hufner, Nucl. Phys. A 608, 356 (1996).

[23] C. Sasaki and K. Redlich, Nucl. Phys. A 832, 62 (2010).

[24] N. Demir and S. A. Bass, Phys. Rev. Lett. 102, 172302 (2009).

[25] R. Marty, E. Bratkovskaya, W. Cassing, J. Aichelin, and H. Berrehrah, Phys. Rev. C 88, 045204 (2013).

[26] P. K. Kovtun, D. T. Son, and A. O. Starinets, Phys. Rev. Lett. 94, 111601 (2005).

[27] J. W. Chen, M. Huang, Y. H. Li, E. Nakano, and D. L. Yang, Phys. Lett. B 670, 18 (2008); J. W. Chen, C. T. Hsieh, and H. H. Lin, ibid. 701, 327 (2011).

[28] M. Buballa, Phys. Rep. 407, 205 (2005); T. Hatsuda and T. Kunihiro, ibid. 247, 221 (1994); S. P. Klevansky, Rev. Mod. Phys. 64, 649 (1992). 
[29] S. Ghosh, Ph.D. thesis, Homi Bhabha National Institute, 2012, http://www.hbni.ac.in/phdthesis/thesis_june2013 /PHYS04200704005_Sabyasachi_Ghosh.pdf

[30] H. A. Weldon, Phys. Rev. D 28, 2007 (1983).
[31] T. Frederico and G. A. Miller, Phys. Rev. D 45, 4207 (1992).

[32] U. G. Meissner, Phys. Rep. 161, 213 (1988).

[33] S. Ghosh and S. Sarkar, Nucl. Phys. A 870, 94 (2011).

[34] S. Ghosh and S. Sarkar, Eur. Phys. J. A 49, 97 (2013). 